PROCEEDINGS OF THE

AMERICAN MATHEMATICAL SOCIETY

Volume 133, Number 9, Pages 2543-2547

S 0002-9939(05)08011-1

Article electronically published on April 8, 2005

\title{
ON FINITE GROUPS ADMITTING A SPECIAL NONCOPRIME ACTION
}

\author{
GÜLIN ERCAN
}

(Communicated by Jonathan I. Hall)

\begin{abstract}
An important result of Turull (1984) is the following:
Let $G A$ be a finite solvable group, $G \triangleleft G A$ and $(|G|,|A|)=1$. Then $f(G) \leq f\left(C_{G}(A)\right)+2 \ell(A)$, where $f$ denotes the Fitting height and $\ell$ denotes the composition length.

The purpose of this work is to give a treatment of the minimal configuration in this framework with additional conditions, yet without the coprimeness condition.
\end{abstract}

Here we will prove (see Theorem 2) the following:

Let $G$ be a finite solvable group and let $\alpha$ be an automorphism of $G$ of order $p$ for some prime $p$. Assume that the orders of elements of $H=G\langle\alpha\rangle$ lying outside of $G$ are not divisible by $p^{2}$. If $C_{\mathcal{S}}(x)$ is nilpotent for any $x \in H-G$ of order $p$ and for any $x$-invariant section $\mathcal{S}$ of $G$, then $f(G) \leq 3$. Furthermore, if the nilpotency condition is replaced by abelianness, then $f(G) \leq 2$.

An immediate consequence of this theorem is a particular case of Turull's result (see also [1] and [4):

Let $G$ be a finite solvable group and let $\alpha$ be an automorphism of $G$ of order $p$ for some prime $p$ where $(|G|,|\alpha|)=1$. If $C_{G}(\alpha)$ is nilpotent, then $f(G) \leq 3$. Furthermore if $C_{G}(\alpha)$ is abelian, then $f(G) \leq 2$.

Although our main purpose is the proof of Theorem 2, and Theorem 1 below makes its appearence as an auxiliary, it should be pointed out that Theorem 1 is of independent interest, too. Theorem 1 is, in its turn, a generalization of the following Lemma.

Lemma ([3] Lemma 1]). Let $G=S T$ be a group where $S \triangleleft G, S$ is a p-group and $T$ is a t-group for distinct primes $p$ and $t$, and let $\alpha$ be an automorphism of $G$ of order $p^{n}$ which leaves $T$ invariant. Assume that $C_{T / T_{0}}(z)=1$, where $T_{0}=C_{T}(S)$ and $z=\alpha^{p^{n-1}}$. Let $V$ be a $k G\langle\alpha\rangle$-module on which $S$ acts faithfully and $k$ is a field of characteristic different from $p$. If $\left[C_{V}(z), C_{S}(z)\right]=1$, then $[S, T]=1$.

Theorem 1. Let $\langle\alpha\rangle$ be a cyclic group of order $p^{n}$ for some prime $p$, and let $G$ be a group acted on by $\langle\alpha\rangle$. Suppose that $S \triangleleft G\langle\alpha\rangle$ is an s-group and $T$ is an $\langle\alpha\rangle$-invariant $t$-subgroup of $G$ for distinct primes $s$ and $t$, such that $[S, T] \neq 1$. Let $V$ be a $k G\langle\alpha\rangle$-module on which $S$ acts faithfully, where $k$ is a field of characteristic

Received by the editors May 17, 2004.

2000 Mathematics Subject Classification. Primary 20D10, 20F28.

Key words and phrases. Noncoprime action, fitting height.

(C)2005 American Mathematical Society Reverts to public domain 28 years from publication 


$$
\begin{aligned}
& \text { not dividing s. Let } z=\alpha^{p^{n-1}} \text {. Then either }\left[C_{V}(z), C_{S}(z)\right] \neq 1 \\
& \quad \text { or }\left[C_{V}(z), C_{T}(z)\right] \neq 1 \text { for some } \bar{x} \in\left(T / T_{0}\right)\langle\alpha\rangle-\left(T / T_{0}\right) \text { of order } p, \\
& \text { or }\left[C_{S}(x), C_{T / T_{0}}(x)\right] \neq 1 \text { for }
\end{aligned}
$$
where $T_{0}=C_{T}(S)$.

Proof. Set $H=G\langle\alpha\rangle$ and use induction on $|H|+\operatorname{dim}_{k} V$. We may assume that $n=1$ and $G=S T$.

(1) $\Phi\left(T / T_{0}\right)=1$ and $\langle\alpha\rangle$ acts irreducibly on $T / T_{0}$.

This is an immediate consequence of induction argument applied to $S T_{1}\langle\alpha\rangle$ on $V$ for a minimal $\langle\alpha\rangle$-invariant subgroup $T_{1} / T_{0}$ of $T / T_{0}$.

(2) $t \neq p$.

Assume the contrary. Then $T / T_{0}$ is centralized by any $1 \neq \bar{x} \in\left(T / T_{0}\right)\langle\alpha\rangle-$ $\left(T / T_{0}\right)$. Let $U$ be an irreducible $T\langle\alpha\rangle$-submodule of $S / \Phi(S)$ on which $T$ acts nontrivially, and let $\bar{t} \in T / T_{0}$ such that $[U, \bar{t}] \neq 1$. Then $C_{U}(\bar{t})=1$. This yields a contradiction as $U=\left\langle C_{U}(a) \mid 1 \neq a \in\langle\bar{t}, \alpha\rangle\right\rangle$ by $([\underline{6}, 5.3 .16])$ and $\left[C_{U}(\bar{x}), T / T_{0}\right]=1$ for any $1 \neq \bar{x} \in\langle\bar{t}, \alpha\rangle-\langle\bar{t}\rangle$.

(3) $S / \Phi(S)$ is an irreducible $T\langle\alpha\rangle$-module with $[S, T]=S,[\Phi(S), T]=1$ and $S$ is special.

Let $S_{1}$ be a normal subgroup of $H$ properly contained in $S$ on which $T$ acts nontrivially. Put $T_{1}=C_{T}\left(S_{1}\right)$. By induction, there exists $\bar{x} \in\left(T / T_{1}\right)\langle\alpha\rangle-\left(T / T_{1}\right)$ such that $\left[C_{S_{1}}(x), C_{T / T_{1}}(x)\right] \neq 1$. As $t \neq p$, this yields that $\left[C_{S_{1}}(x), C_{T / T_{0}}(x)\right] \neq 1$ which is not the case. Thus $T\langle\alpha\rangle$ acts irreducibly on $S / \Phi(S),[S, T]=S,[\Phi(S), T]=$ 1 and $S$ is special.

(4) $[T, \alpha]=1$.

Assume the contrary. Then $C_{T / T_{0}}(\alpha)=1$ and so $C_{S / \Phi(S)}(\alpha) \neq 1$. Now $s \neq p$, because otherwise $\left[C_{V}(\alpha), C_{S}(\alpha)\right] \neq 1$ by the Lemma.

Let $M$ be an irreducible $S T\langle\alpha\rangle$-submodule of $V$ on which $S$ acts nontrivially. Then $[M, S]=M$ and so $[M, T] \neq 1$. Set $\bar{S}=S / C_{S}(M)$. By Clifford's theorem aplied to $\bar{S} T$ on $M$, we have that $M=W_{1} \oplus \cdots \oplus W_{r}$, where the $W_{i}$ 's are homogeneous $\bar{S} T$-modules. Here $N_{\langle\alpha\rangle}\left(W_{1}\right)=N_{\langle\alpha\rangle}\left(W_{i}\right)$ for each $i=1, \cdots, r$ and so either $r=1$ or $r=p$. If the latter holds, then $\left[W_{i}, C_{\bar{S}}(\alpha)\right]=1$ for each $i$, because $\left[C_{M}(\alpha), C_{S}(\alpha)\right]=1$ and $s \neq p$. It follows that $C_{S}(\alpha) \leq C_{S}(M)$ and so $C_{S}(\alpha) \leq \Phi(S)$ which is not the case. Thus $M$ is a homogeneous $\bar{S} T$-module, i.e. $M=M_{1} \oplus \cdots \oplus M_{i}$ with $M_{i} \cong M_{1}$ irreducible $\bar{S} T$-modules.

If $\bar{S}$ is nonabelian, then $[\Phi(\bar{S}), \alpha]=1$ and so $C_{M}(\alpha) \leq C_{M}(\Phi(S))=1$. This shows that char $k \neq p$. Observe that $[\bar{S}, \alpha] \neq 1$, because otherwise $[\bar{S}, T]=1$ by the three subgroup lemma. By [5] applied to the action of both $[\bar{S}, \alpha]\langle\alpha\rangle$ and $T\langle\alpha\rangle$ on $M$, we conclude that $s=2=t$, which is impossible.

Thus $\bar{S}$ is abelian. The number of homogeneous components of $\left.M_{1}\right|_{S}$ is a power of $t$ and so the number of homogeneous components of $\left.M\right|_{S}$ is also a power of $t$. Since $t \neq p, \alpha$ fixes a homogeneous component $W$ of $\left.M\right|_{\bar{S}}$. If $U$ is a homogeneous component of $\left.M\right|_{S}$ which is $\alpha$-invariant and different from $W$, then $W=U^{a}$ for some $a \in T\langle\alpha\rangle$. Now $[a, \alpha] \in N_{T}(W)$ and so $1 \neq C_{T / N_{T}(W)}(\alpha) \cong C_{\left(T / T_{0}\right) /\left(N_{T}(W) / T_{0}\right)}(\alpha)$, i.e. $C_{T / T_{0}}(\alpha) \neq 1$, which is not the case. Thus $\alpha$ fixes exactly one homogeneous component $W$ of $\left.M\right|_{\bar{S}}$. Observe that either $N_{T}(W)=T$ or $N_{T}(W) \leq T_{0}$. If the first holds, then $[[\bar{S}, T], W]=[\bar{S}, W]=1$ implying that $C_{M}(\bar{S}) \neq 1$, which is not the case. Hence $N_{T}(W) \leq T_{0}$. Also note that $[\bar{S}, \alpha] \neq 1$, because otherwise $[\bar{S}, T]=1$ by the three subgroup lemma. Now $[[\bar{S}, \alpha], W]=1$, and so there exists 
a homogeneous component $U$ of $\left.M\right|_{\bar{S}}$ such that $U \neq U^{\alpha}$. Here note that

$$
C_{\bar{S}}(\alpha) \leq \operatorname{Ker}(\bar{S} \text { on } U),
$$

as $\left[C_{U}(\alpha), C_{S}(\alpha)\right]=1$, and so

$$
C_{\bar{S}}(\alpha) \cap \operatorname{Ker}(\bar{S} \text { on } W) \leq \operatorname{Ker}(\bar{S} \text { on } M)=1 .
$$

Then $C_{\bar{S}}(\alpha) \cap\left\langle C_{\bar{S}}(\alpha)^{\bar{t}} \mid 1 \neq \bar{t} \in \bar{T}\right\rangle=1$, where $\bar{T}=T / C_{T}(M)$ and so $C_{\bar{S}}(\alpha)^{\bar{x}} \cap$ $\left\langle C_{\bar{S}}(\alpha)^{\bar{t}} \mid \quad \bar{x} \neq \bar{t}\right\rangle=1$. Now $\sum_{\bar{t} \in \bar{T}} C_{\bar{S}}(\alpha)^{\bar{t}}=\bigoplus_{\bar{t} \in \bar{T}} C_{\bar{S}}(\alpha)^{\bar{t}}=\bar{S}$ since $\bar{S}$ is an irreducible $T\langle\alpha\rangle$-module. It follows that $|\bar{S}|=\left|C_{\bar{S}}(\alpha)\right|^{|\bar{T}|}$. On the other hand $\left[\bar{S},\left[\bar{T} / \bar{T}_{0}, \alpha\right]\right]=\bar{S}$ and so $|\bar{S}|=\left|C_{\bar{S}}(\alpha)\right|^{p}$ by Lemma 4.5 in [7]. As $t \neq p$, we get a contradiction. Therefore $\left[T / T_{0}, \alpha\right]=1$, i.e. $C_{T}(\alpha) T_{0}=T$. By induction we see that $C_{T}(\alpha)=T$.

(5) $[S, \alpha]=S$ and so $s \neq p$.

$[S, \alpha]$ is either trivial or the whole of $S$. If it is trivial, then $[S, T]=1$ as $\left[C_{S}(\alpha), C_{T / T_{0}}(\alpha)\right]=1$, a contradiction.

(6) $S$ is abelian.

Assume the contrary. Then $1 \neq \Phi(S)=Z(S)$. Let $M$ be an irreducible $S T\langle\alpha\rangle$ submodule of $V$ on which $\Phi(S)$ acts nontrivially. Set $\bar{S}=S / C_{S}(M)$. We consider $\left.M\right|_{\bar{S} T}=W_{1} \oplus \cdots \oplus W_{r}$, where $W_{i}$ 's are homogeneous $\bar{S} T$-components of $M$. If $r=p$, then $\left[W_{i}, T\right]=1$ for each $i$, as $\left[C_{M}(\alpha), T\right]=1$, and so $[M, T]=1$, which is not the case. Then $r=1$. It follows that $[\Phi(\bar{S}), \alpha]=1$ implying that $C_{M}(\alpha) \leq$ $C_{M}(\Phi(\bar{S}))=1$. If $\Phi(\bar{S})$ is not cyclic, then there exists $1 \neq a \in \Phi(\bar{S})$ such that $C_{M}(a) \neq 1$, by $([6,5.3 .16])$, implying that $C_{\bar{S}}(M) \neq 1$, a contradiction. Hence $\Phi(\bar{S})$ is cyclic and so $\bar{S}$ is estraspecial, where $|\bar{S}|=2^{2 n+1}$ and $p=2^{n}+1$ for some $n \geq 1$, by [5].

By [6, 5.5.2], the number of distinct cyclic subgroups of order 4 in $\bar{S}$ is

$$
\frac{1}{2}\left(2^{2 n} \mp(-2)^{n}\right) \text {. }
$$

Since each cyclic group of order 4 contains two elements of order 4 , and distinct cyclic subgroups of order 4 have no element of order 4 in common, there are $2^{2 n} \mp$ $(-2)^{n}=2^{n}\left(2^{n} \mp 1\right)$ elements of order 4 in $\bar{S}$. As $T\langle\alpha\rangle$ acts irreducibly on $\bar{S} / \Phi(\bar{S})$ and $[\bar{S}, T]=\bar{S}$, we have $C_{\bar{S}}(T) \leq \Phi(\bar{S})$. It follows that $C_{\bar{S}}(T)=\Phi(\bar{S})$, since $[\Phi(\bar{S}), T]=1$. Now $\Phi(\bar{S})$ contains no element of order 4 , since it is cyclic of order 2. Thus $T\langle\alpha\rangle$ permutes the elements of $\bar{S}$ of order 4 , without fixing any, in orbit of length $|(T\langle\alpha\rangle) /(\Phi(T))|=t p$. Therefore $t p$ divides $2^{n}\left(2^{n} \pm 1\right)$. But as $t \neq s=2$ and $p=2^{n}+1, t p$ divides $2^{n}+1=p$ which yields that $t=1$, a contradiction.

(7) Finally, let $M$ be an irreducible $S T\langle\alpha\rangle$-submodule of $V$ on which $S$ acts nontrivially. Set $\bar{S}=S / C_{S}(M)$. Let $\Omega=\left\{W_{1}, \cdots, W_{r}\right\}$ be the set of all homogeneous $\bar{S}$-components of $M$. Since $[S, \alpha]=S$, no $W_{i}$ is $\alpha$-invariant. Because otherwise as $\left[S, W_{i}\right]=1$ for each $i$, we have $C_{M}(S) \neq 1$, a contradiction.

Let $\mathcal{O}=\left\{W, W^{\alpha}, \cdots, W^{\alpha^{p-1}}\right\}$ be an $\alpha$-orbit. Set $\bar{T}=T / C_{T}(M)$ and $X=$ $\bigoplus_{i=0}^{p-1} W^{\alpha^{i}}$. As $\left[C_{X}(\alpha), T\langle\alpha\rangle\right]=1$, we have $\left[W, N_{T\langle\alpha\rangle}(W)\right]=1$. Let $t \in T$. If $Y=X^{t}$, then $C_{Y}(\alpha)=C_{X}(\alpha)^{t}=C_{X}(\alpha)$ and so $X \cap Y \neq 0$, i.e. $X=Y$. Hence $T$ acts on $\mathcal{O}$ and $\mathcal{O}=\Omega$. This gives that $p=|\Omega|=\left|T\langle\alpha\rangle: N_{T\langle\alpha\rangle}(W)\right|$. Then $N_{T\langle\alpha\rangle}(W)=T$ because $T$ is the unique subgroup of $T\langle\alpha\rangle$ of index $p$. This yields that $[W, T]=1$ and so $[M, T]=1$, a contradiction which completes the proof of Theorem 1. 
As a consequence of Theorem 1, we have

Theorem 2. Let $G$ be a finite solvable group and let $\alpha$ be an automorphism of $G$ of order $p$ for some prime $p$. Assume that the orders of elements of $H=G\langle\alpha\rangle$ lying outside $G$ are not divisible by $p^{2}$. If $C_{\mathcal{S}}(x)$ is nilpotent for any $x \in H-G$ of order $p$ and for any $x$-invariant section $\mathcal{S}$ of $G$, then $f(G)$ is at most 3 . Furthermore, if the nilpotency condition is replaced by abelianness, then $f(G) \leq 2$.

Proof. Let $H=G\langle\alpha\rangle$ be a minimal counterexample to the theorem. We may assume that $f(G)=4$. Then by Lemma 1 in [2] there exist subgroups $C_{i}$ of $G$ and subgroups $D_{i} \triangleleft C_{i}$ for $i=1,2,3,4$ and an element $x \in H-G$ of order $p$ such that the following are satisfied:

(i) $C_{i}$ is a $p_{i}$-subgroup for some prime $p_{i}$, i.e. $\pi\left(C_{i}\right)=\left\{p_{i}\right\}$ for any $i$ and $p_{i} \neq p_{i+1}$ for $i=1,2,3$.

(ii) $C_{i}$ and $D_{i}$ are $\left(\prod_{j>i} C_{j}\right)\langle\alpha\rangle$-invariant for any $i$.

(iii) $\bar{C}_{i}=C_{i} / D_{i}$ is a special group on the Frattini factor group of which $\left(\prod_{j>i} C_{j}\right)\langle\alpha\rangle$ acts irreducibly and $C_{i+1}$ acts trivially on $\Phi\left(\bar{C}_{i}\right)$ for any $i$.

(iv) $\left[C_{i}, C_{i+1}\right]=C_{i}$ for $i=1,2,3$.

(v) $C_{C_{i+1}}\left(\bar{C}_{i} / \Phi\left(\bar{C}_{i}\right)\right)=C_{C_{i+1}}\left(\bar{C}_{i}\right)$ is contained in $\Phi\left(C_{i+1} \bmod D_{i+1}\right)$ for $i=$ $1,2,3$.

(vi) $\left[C_{j}, C_{i}\right]$ is not contained in $\Phi\left(C_{j} \bmod D_{j}\right)$ for any $i=2,3,4$ and any $1 \leq$ $j<i$.

Put $K=C_{1} C_{2} C_{3} C_{4}$. Now $K\langle x\rangle$ satisfies the hypothesis of the theorem.

Applying Theorem 1 to the action of $\bar{C}_{3} C_{4}\langle x\rangle$ on the Frattini factor group $\tilde{C}_{2}$ of $\bar{C}_{2}$ we see that $\left[C_{\tilde{C}_{2}}(x), C_{C_{4}}(x)\right] \neq 1$ with the requirement $\pi\left(C_{2}\right)=\pi\left(C_{4}\right)$. Also applying Theorem 1 to the action of $\bar{C}_{2} C_{3}\langle x\rangle$ on $C_{1}$ we see that $\left[C_{C_{1}}(x), C_{C_{3}}(x)\right] \neq 1$ with the requirement $\pi\left(C_{1}\right)=\pi\left(C_{3}\right)$. Now $D_{4}=C_{C_{4}}\left(\bar{C}_{2}\right)$ and so $C_{C_{4}}(x) \not \leq D_{4}$, i.e. $\left[\bar{C}_{4}, x\right]=1$ This forces that $C_{\bar{C}_{3}}(x) \leq \Phi\left(\bar{C}_{3}\right)$, because otherwise $\left[\bar{C}_{3} \bar{C}_{4}, x\right]=1$, which is not the case. Then $C_{\bar{C}_{3}}(x) \leq Z\left(\bar{C}_{3} C_{4}\langle x\rangle\right)$ and so $C_{\tilde{C}_{2}}\left(C_{\bar{C}_{3}}(x)\right)$ is either trivial or $\tilde{C}_{2}$. If it is trivial, then $C_{\tilde{C}_{2}}(x)=1$, which is not the case. Hence $C_{\bar{C}_{3}}(x)=1$, i.e. $C_{C_{3}}(x) \leq D_{3}=C_{C_{3}}\left(C_{1}\right)$ as $\pi\left(C_{1}\right)=\pi\left(C_{3}\right)$, a contradiction. This completes the proof of the first claim.

The last claim can be easily shown by an application of Theorem 1 to $C_{1} C_{2} C_{3}\langle x\rangle$, where $C_{i}$ are subgroups of $H$ and $D_{i} \triangleleft C_{i}, i=1,2,3$, satisfying (i)-(vi).

Corollary. Let $G$ be a finite solvable group and let $\alpha$ be an automorphism of $G$ of order $p$ for some prime $p$ where $(|G|,|\alpha|)=1$. If $C_{G}(\alpha)$ is nilpotent, then $f(G) \leq 3$. Furthermore if $C_{G}(\alpha)$ is abelian, then $f(G) \leq 2$.

\section{REFERENCES}

1. Asar, A.O.: Automorphism of prime order of soluble groups whose subgroups of fixed points are nilpotent. Journal of Algebra 88, 178-189 (1984). MR0741938 (85k:20069)

2. Ercan G., Güloğlu, İ.: On the Fitting length of $H_{n}(G)$. Rend. Sem. Mat. Univ. Padova, 89 (1993). MR 1229051 (94f:20035)

3. Ercan, G, Güloğlu, I.: On finite groups admitting a fixed point free automorphism of order $p q r$, J. Group Theory 7 (2004), no. 4, 437-446. MR2080444

4. Feldman, A.: Fitting height of soluble groups admitting an automorphism of prime order with abelian fixed point subgroup, Journal of Algebra 68, 97-108 (1981). MR0604296 (83b:20021)

5. Gagola, S., Jr.: Solvable groups admitting an almost fixed point free automorphism of prime order. Illinois J. Math. 22, 191-207 (1978). MR0473007 (57:12686) 
6. Gorenstein, D.: Finite Groups, New York (1968). MR0231903 (38:229)

7. Hartley, B., Turau, V.: Finite solvable groups admitting an automorphism of prime power order with few fixed points. Math. Proc. Camb. Phil. Soc., 431-441 (1987). MR0906617 (88i:20041)

8. Turull, A.: Fitting height of groups and of fixed points. Journal of Algebra 86, 555-566 (1984). MR0732266 (85i:20021)

Department of Mathematics, Middle East Technical University, 06531, Ankara, TURKEY 